\title{
Reflections from a NMOSD case with serum AQP4-Ab negativity but CSF positivity: narrative review of how to interpret AQP4-Ab test results
}

\author{
Qun Xue ${ }^{1,2,3}$, Shugang Cao ${ }^{1,4}$, Qianyun Rui ${ }^{1}$ \\ ${ }^{1}$ Department of Neurology, the First Affiliated Hospital of Soochow University, Suzhou, China; ${ }^{2}$ Jiangsu Institute of Clinical Immunology, the First \\ Affiliated Hospital of Soochow University, Jiangsu Key Laboratory of Clinical Immunology, Soochow University, Suzhou, China; ${ }^{3}$ Suzhou Clinical \\ Medical Centre of Neurological Disorders, Suzhou, China; ${ }^{4}$ Department of Neurology, the Affiliated Hefei Hospital of Anhui Medical University, \\ Hefei, China \\ Contributions: (I) Conception and design: Q Xue; (II) Administrative support: Q Xue; (III) Provision of study materials or patients: S Cao, Q Rui; (IV) \\ Collection and assembly of data: S Cao, Q Rui; (V) Data analysis and interpretation: All authors; (VI) Manuscript writing: All authors; (VII) Final \\ approval of manuscript: All authors. \\ Correspondence to: Qun Xue. Department of Neurology, the First Affiliated Hospital of Soochow University, Suzhou, China. Email: qxue_sz@163.com.
}

\begin{abstract}
Neuromyelitis optica spectrum disorder (NMOSD) is an inflammatory demyelinating disease of the central nervous system. The differential diagnosis of NMOSD in clinical practice is often challenging despite the phenotypical and serological characteristics of the disease. The discovery of anti-aquaporin- 4 antibody (AQP4-Ab) enabled clinicians to diagnose NMOSD relatively earlier and more easily, as the AQP4-Ab can mediate the pathogenesis of NMOSD. Testing for AQP4-Ab in the serum of patients can play a crucial role in the diagnosis of NMOSD. Three-quarters of patients with NMOSD have serum immunoglobulin-G (IgG) autoantibodies to the AQP4 channel. Nevertheless, the test results for AQP4-Ab can be affected by several factors, such as assay methods, clinical stages, the types of treatment, sample status, and pre-test error, among others. In patients with seronegative NMOSD, it would be better to test serum and CSF AQP4-Ab together to improve the positive rate, especially when NMOSD is highly suspected. This article aims to update readers on the recent developments in AQP4-Ab testing and how to interpret the results of the AQP4-Ab test.
\end{abstract}

Keywords: Neuromyelitis optica spectrum disorder (NMOSD); autoantibody to aquaporin-4 (AQP4-Ab); cellbased assay (CBA)

Submitted May 20, 2020. Accepted for publication Jul 02, 2021. Published online Jul 14, 2021.

doi: 10.21037/atm-20-4110

View this article at: https://dx.doi.org/10.21037/atm-20-4110

\section{Introduction}

Idiopathic inflammatory demyelinating diseases (IIDDs) of the central nervous system (CNS) mainly comprise multiple sclerosis (MS), neuromyelitis optica spectrum disorder (NMOSD), and acute disseminated encephalomyelitis (ADEM). These diseases usually show specific differences in the age of onset, clinical courses, magnetic resonance imaging (MRI) findings, cerebrospinal fluid (CSF) features, treatment, and morbidity. However, overlapping features in clinical, MRI, and serum/CSF findings often make their diagnosis difficult, especially at the first demyelinating event. Thus, the definite diagnosis at early stage is of greatest importance in anticipating the potential further disease course and in determining the methods of treatment. NMOSD is not always a relapsing disease but with a high risk of morbidity and disability (1). NMOSD patients benefit specifically from B-cell/antibody targeting drugs (2), but may worsen with some disease modifying therapies (DMTs) approved for MS. The seminal finding of specific pathogenic antibodies against aquaporin-4 (AQP4) separated 
NMOSD as a disease entity from MS (3). Three-quarters of patients with NMOSD have serum immunoglobulin-G (IgG) autoantibodies to the aquaporin-4 protein. This biomarker is so important that the new diagnosis criterion recommended the classification of NMOSD into two types: the AQP4 antibody (AQP4-Ab) positive and negative subtypes (1). Nevertheless, the test results for AQP4-Ab can be affected by several factors, such as assay methods, clinical stages, the types of treatment, sample status, and pre-test error, among others. This review aims to update readers on the recent developments in AQP4-Ab testing and how to interpret the results of the AQP4-Ab test. We present the following article in accordance with the Narrative Review reporting checklist (available at https://atm.amegroups.com/ article/view/10.21037/atm-20-4110/rc).

\section{A difficult scenario}

A 45-year-old female has been admitted to our hospital three times. For the first time, she presented for an inability to defecate and urinate, accompanied by weakness of the lower limbs and slurred speech. Five years before, she had experienced repeated pain in the shoulder, neck and ear without any predisposing factors, followed by double vision without dizziness, headache, nausea and vomiting, which could be relieved spontaneously. The pain and double vision reappeared once or twice a year, lasting for several days each episode. Brain MRI revealed abnormal signs in the bilateral frontal lobes and corpus callosum, and left basal ganglia and temporal lobe (Figure $1 A, 1 B$ ), but spinal cord MRI showed no abnormality. The somatosensory evoked potential (SEP) was normal. The visual evoked potential (VEP) showed prolonged P100 wave latencies bilaterally, and P100 wave amplitudes reduced to $40 \%$ of the normal value on the left and $50 \%$ of the normal value on the right. Her thyroid function and tumor markers were normal. Her antinuclear antibodies (ANA) profile (including anti-SSA antibody, anti-SSB antibody, and anti-ds-DNA antibody, etc.), anti-neutrophil cytoplasmic antibodies (ANCA), anti-cardiolipin antibodies (ACA), and T-SPOT test were all negative. Serum AQP4-Ab test results were as follows: cell-based assay (CBA), negative (1:8) (conducted by Jiangsu Institute of Clinical Immunology, the First Affiliated Hospital of Soochow University in Appendix 1); enzyme-linked immunosorbent assay (ELISA) (the kit from RSR, UK), $0.91 \mathrm{U} / \mathrm{mL}$ (normal range: $<3 \mathrm{U} / \mathrm{ml}$ ); and indirect immunofluorescence (IIF) testing [conducted by Euroimmun Medical Diagnostics (China) Co., Ltd.,
Hangzhou], both AQP4-Ab and myelin oligodendrocyte glycoprotein antibodies (MOG-IgG) were negative. The results of CSF test revealed CSF protein $1.113 \mathrm{~g} / \mathrm{L}$ (normal range, $0.15-0.45 \mathrm{~g} / \mathrm{L}$ ), white blood cell (WBC) count $48 \times 10^{6} / \mathrm{L}$ (normal range, $0-5 \times 10^{6} / \mathrm{L}$ ), CSF IgG index 0.76 (normal range, $\leq 0.7$ ), and no tumour cells. Oligoclonal band (OB) was negative in both serum and CSF. The albumin $\mathrm{CSF} /$ serum quotient (Q-Alb) was 16.05 (normal range, $<16)$. PET-CT showed no prominent increased ${ }^{18} \mathrm{~F}-\mathrm{FDG}$ uptake in the whole brain and spinal cord. The patient was treated with $500 \mathrm{mg}$ methylprednisolone (MPDN) by intravenous infusion each day for five days, which was gradually decreased. A repeated MRI of the brain and spinal cord after two weeks revealed no obvious changes.

Seven months later, she was hospitalized for the second time. One and a half months before admission, she suffered from optical neuritis (mainly blurred vision in her right eye) and was hospitalized in a local hospital, and repeated enhanced MRI of the brain showed abnormal signals in the right optical nerve without the involvement of the optic chiasm, and the abnormal signal in the brain disappeared almost compared with the previous MR (Figure 1C). Optical coherence tomography (OCT) examination showed that the thickness of the macula was significantly reduced bilaterally and was more severe in the right eye. The VEP was not elicited in the right eye, and the P100 wave latency was prolonged in the left eye. She was treated with $120 \mathrm{mg} /$ day MPDN intravenously for five days, and her condition gradually improved. After this admission, by excluding the possibility of tumors and infectious diseases such as tuberculosis (TB) and hepatitis B, she was treated with 600 mg Rituximab (RTX). The proportion of peripheral CD3 ${ }^{-}$ CD $19^{+}$B cell was reduced to $0 \%$ one week after RTX treatment.

The patient was admitted for the third time four months later. She felt fatigue and paroxysmal throbbing pain in her ribs, followed by slurred speech. In addition, physical examination showed that her visual acuity decreased significantly in the left eye. Before this admission, she underwent urinary catheterization in the local hospital. The proportion of $\mathrm{CD}^{-} \mathrm{CD} 19^{+} \mathrm{B}$ cells was $0.28 \%$ and that of $\mathrm{CD} 19^{+} \mathrm{CD} 27^{+}$memory B cells was $0.1 \%$. The CSF test showed protein $0.730 \mathrm{mg} / \mathrm{L}$ and $\mathrm{WBC}$ count $12 \times 10^{6} / \mathrm{L}$. Cryptococcus test and TB antibody test were both negative. Repeated enhanced brain MRI revealed that the signs in bilateral paraventricular white matter were worse than five months before (Figure 1D). No abnormality was found in non-enhanced and enhanced MRI of the whole spinal cord. 

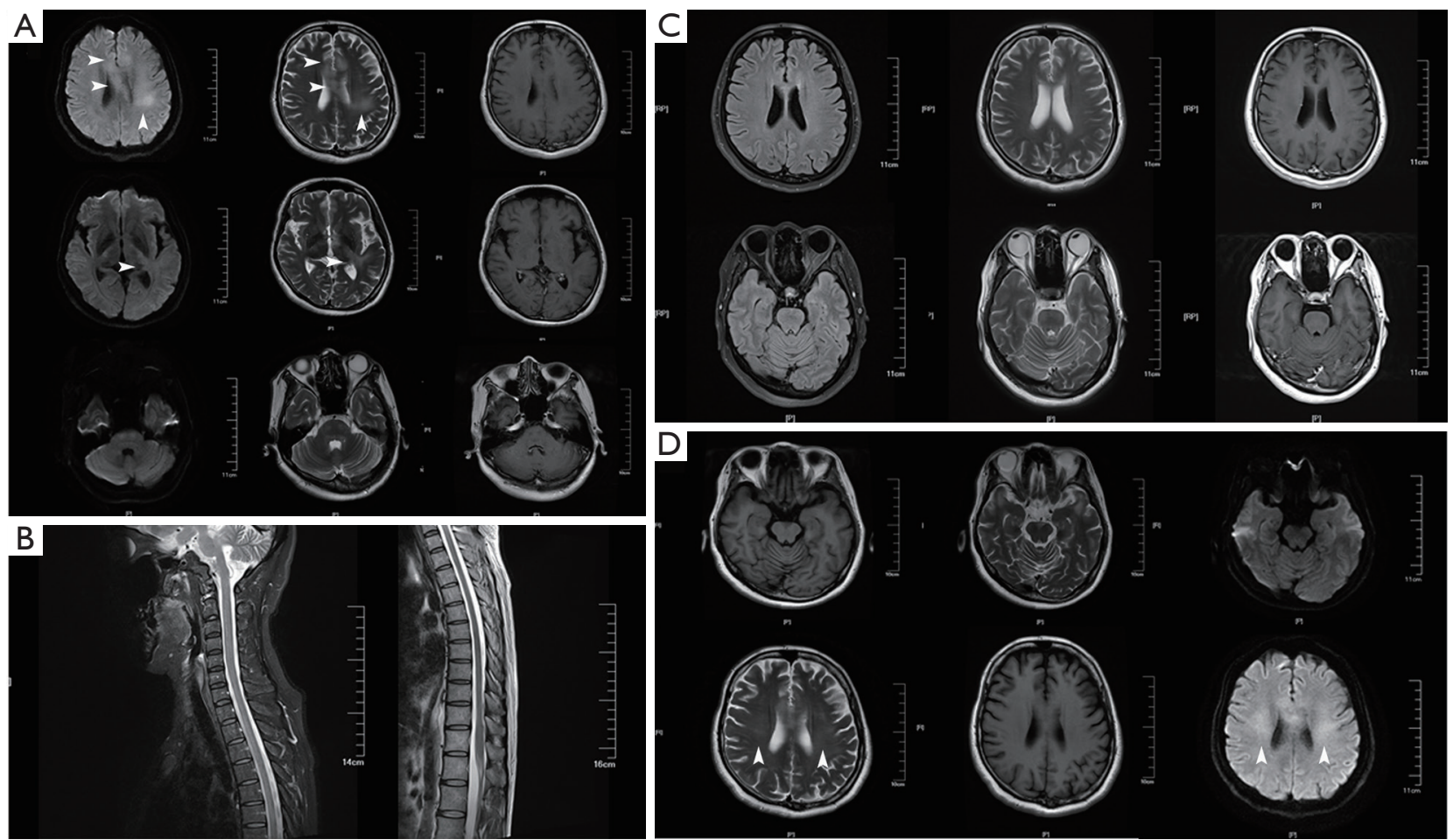

Figure 1 Brain and spinal cord MRI results. (A) Brain MRI on July 6, 2017. It showed abnormal signs in the bilateral frontal lobes and corpus callosum, left basal ganglia and left temporal lobe (white arrows). (B) T2-weighted MRI of the spinal cord found no abnormal signal. (C) Brain MRI on January 8, 2018. It showed abnormal signals in the right optical nerve but improved signals in the left centrum semiovale. (D) Brain MRI on June 12, 2018. It revealed that the signs in the bilateral paraventricular white matter (white arrows) had progressed over the past 5 months, suggesting demyelination. MRI, magnetic resonance imaging.

Repeat serum AQP4-Ab showed ELISA $2.04 \mathrm{U} / \mathrm{mL}$ and CBA negative (1:16), but CSF AQP4-Ab revealed ELISA $4.72 \mathrm{U} / \mathrm{mL}$ and CBA positive $(1: 1,000)$. To avoid severe infection, she was only given $80 \mathrm{mg} /$ day MPDN for seven days. Then MPDN was gradually decreased. One and half months later, her condition was stable, and repeated serum and CSF AQP4-Ab tests were both negative. Azathioprine plus steroid was used for maintenance treatment. Figure 2 summarizes the whole course of the patient. Ten months later, her ANA profile was negative, and repeated serum and CSF AQP4-Ab tests using CBA were still both negative. The abnormality on the brain MRI improved compared to the last MRI scan. The long-term follow-up is still ongoing.

\section{Discussion on the role of AQP4 antibody in the diagnosis and management}

In this case, at the first hospital visit, infections or tumours were ruled out via systematic examination. Short-term relapse occurred after an interval. There were some similarities with MS, such as being female, repeated fatigue, pain, bowel and bladder disturbances, and increased IgG index. However, the MRI results did not support MS. Because AQP4-Ab in serum and CSF was negative, the patient was initially diagnosed with IIDDs. No MDT was used. Six months later, when she relapsed with blindness in the right eye, the long optic nerve damage on MRI grew up to $1 / 2$ in length, and the VEP and OCT results showed severe damage, supporting optic neuritis $(\mathrm{ON})$. The clinical course seemed to meet the 2015 IPND diagnostic criteria for NMOSD (The lesions of this patient involved the optic nerve, periventricular tissues and corpus callosum, etc.) (1). Nevertheless, we could still not confidently make the diagnosis of NMOSD due to the negative AQP4-Ab. Since this patient had frequent attacks and severe neurological damage, RTX was used to reduce recurrence after ruling out tumours, infections and other inflammation-related diseases. The recurrence 4 months later led us to go back 


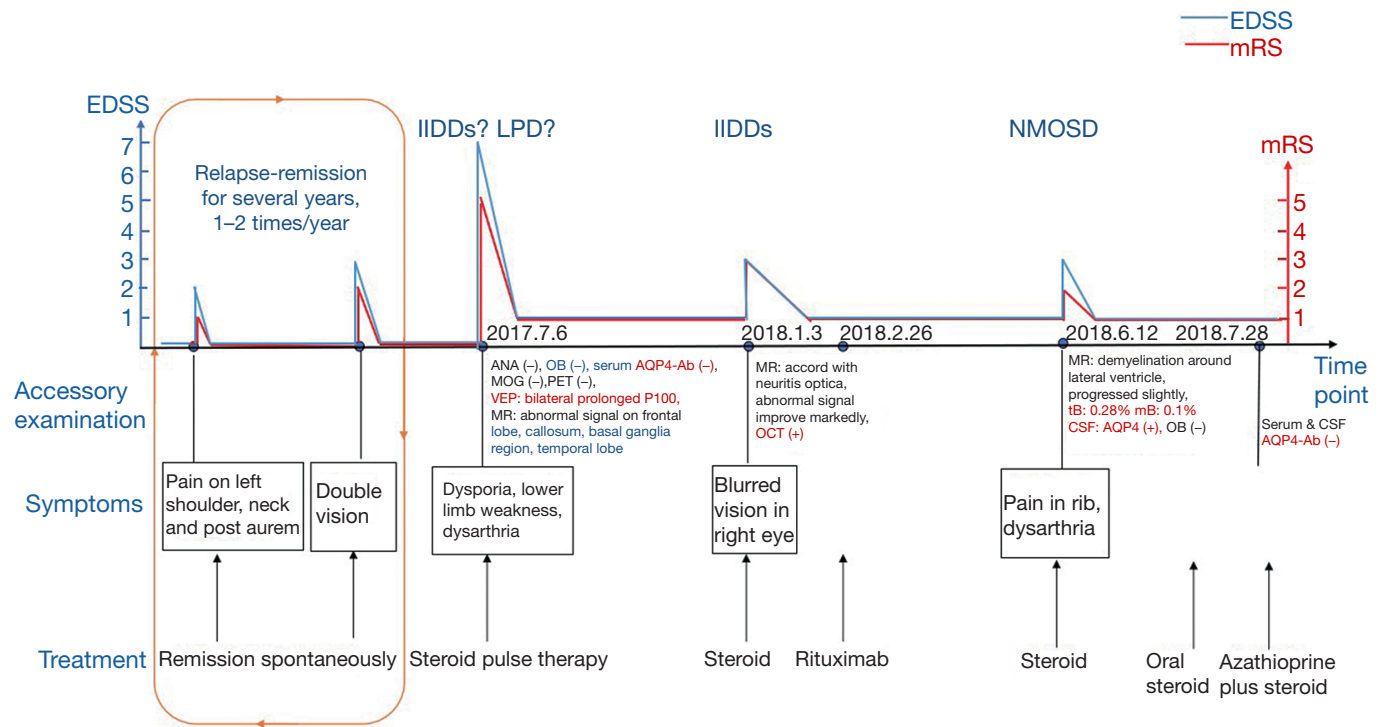

Figure 2 The clinical course of the case was based on the mRS and treatments. IIDDs, idiopathic inflammatory demyelinating diseases; LPD, lymphoproliferative disease; NMOSD, neuromyelitis optica spectrum disorder; AQP4-Ab, aquaporin-4 antibody; OCT, optical coherence tomography; VEP, visual evoked potential; OB, oligoclonal band; mRS, modified Rankin scale; EDSS,Expanded Disability Status Scale; MR, magnetic resonance; ANA, antinuclear antibodies; MOG, myelin oligodendrocyte glycoprotein; PET, positron emission tomography; CSF, cerebrospinal fluid.

to systematic screening to rule out differential diagnosis. Once again, we checked serum and CSF AQP4-Ab using CBA and ELISA in our laboratory and in Euroimmun (Hangzhou). This time, we were surprised to find that both the results from two labs showed positive AQP4-Ab in CSF, but negative AQP4-Ab in serum. The final diagnosis of this case was consistent with NMOSD with AQP4-Ab positivity, though there are still some questions worth discussing.

Earlier repeated AQP4-Ab detection might help us to make the diagnosis in this case. Usually, the positive rate of serum AQP4-Ab is higher than that in CSF (4). However, in this case, the AQP4-Ab was only detectable in CSF. Although the ELISA result of AQP4-Ab was positive, the titre was not high. On the contrary, the CBA titre was very high. The two results were not consistent. Are the results trustable? How do we interpret the AQP4-Ab results?

\section{Key points for interpreting the AQP4-Ab results}

AQP4-Ab test results might be affected by some factors such as detection methods, sample pre-test state including sampling time, sample transportation, storage, repeated freeze-thaw, etc., clinical conditions, and so forth. When interpreting the test result, the following factors should be considered if the result did not match the clinical and imaging manifestations.

\section{AQP4-Ab detection methods}

The AQP4-Ab detection methods currently include IIF, ELISA, CBA, and fluorescence activated cell sorting (FACS).

(I) The IIF was the first assay method used to identify NMO-IgG (5) and can be useful as a screening tool for diverse antibodies against the CNS antigens, including $\mathrm{AQP} 4-\mathrm{Ab}$, with a relatively low cost and low sensitivity $(5-73 \%)$, although the specificity $(91-100 \%)$ is acceptable (5-8).

(II) ELISA may quantify the titre of AQP4-Ab, but has a relatively poor diagnostic accuracy due to the low sensitivity of $71 \%(7,9-11)$. The normal value of AQP4-Ab in our lab is less than $3 \mathrm{U} / \mathrm{mL}$, following the manufacturer's operating manual. CBA was recommended strongly according to the international guidelines for diagnosing NMOSD (1). Because ELISA is more convenient to follow-up the change of the titer and consistency analysis of the disease state in each case, we adopted both CBA and 
ELISA (RSR, UK) to detect AQP4-Ab.

(III) Fixed-CBA using a commercial kit coated with prefixed cells (expressing human M1-AQP4) is the most widely used currently, as it is ready to use and has a relatively good accuracy (12). However, due to the pre-fixation of the AQP4-expressing cells, the three-dimensional structure of the M1-AQP4 isoform might be damaged, and a false-negative result might occur $(13,14)$. The live-CBA using live cells expressing human M23-AQP4 seems to have a higher accuracy than the fixed-CBA, but it demands a high level of technical expertise and is time-consuming, which limits its use to some specialized centres $(8,15)$. Therefore, if the AQP4-Ab assay results, performed with the fixedCBA results, are negative, but the clinical and/or radiological manifestations are very supportive of NMOSD, it would be reasonable to retest their samples with the live-CBA (the cells transfected with the M23 subunit) or the CBA with both M1 and M23 transfected (in our hospital).

(IV) The FACS assay, using free-floating live cells expressing human AQP4 transfected with the M23 subunit, might have a higher sensitivity than the fixed-CBA or even the live-CBA. The accuracy varied greatly according to the methodological details and the experience of the examiners, which limited the use of the FACS assay, though it has the advantages of its semi-quantitative results and a diagnostic threshold (8,11,15-17).

At present, most research institutions adopt M23-AQP4 transfected cells in the CBA. When adopting M23-AQP4 transfected cells, special procedures should be followed for the storage of slides and the preservation of cell lines used for fluorescence reaction detection. Therefore, long-term culture requires more labour, material resources and costs. In view of this, our laboratory constructed an HEK293T cell line transfected with lentivirus containing M1 and M23AQP4 sequence. Our CBA method can detect antibodies to the $M 1$ and M23 subunits, which is different from the other CBA method commonly used to detect only M23 subunit antibodies $(13,18)$.

\section{Sample pre-test state}

Some pre-test factors may affect the result, including sampling time, sample transportation and storage, repeated freeze-thaw, etc.
(I) If the time of sample collection is immediately after or during plasmapheresis, high-dose corticosteroids, or B-cell-depleting treatment (e.g., rituximab) as well as during the remission stage, it might have lower titres of AQP4-Ab or the AQP4Ab even turns negative (19).

(II) The pre-test factors caused by poor transportation and storage conditions, for instance haemolysis or delayed measurement of the blood samples, should not be ignored.

(III) At present, the effect of repeated freeze-thaw cycles on the detection of AQP4-Ab in serum and CSF remains controversial. The study by Jarius et al. (20) suggested that AQP4-Ab were relatively stable over a period of at least 8 days at room temperature or $4{ }^{\circ} \mathrm{C}$ and that AQP4-Ab levels were not affected to any significant degree by repeat freeze-thaw cycles. However, for most ELISA kits, the serum samples can be kept at $4{ }^{\circ} \mathrm{C}$ for at least 5 days before testing. If it would be tested one week later, the samples should be frozen for later detection. When the serum is thawed, the sample should be adequately mixed for the protein well-distributed. Repeated freeze-thaw cycles would decrease the titres of the antibodies. The mechanical shear force produced by repeated freezing and thawing would destroy the protein and other molecules in the specimen, thus causing false-negative results. According to the study by Yang et al. (21), compared with the fresh serum samples, the AQP4-Ab titers of serum samples stored for a long time were affected to some extent after several freeze-thaw cycles. Although it had little effect on the positive rate, the fluorescence intensity of detection is obviously decreased. After 5 times of repeated freezing and thawing, the detection effect of AQP4-Ab in CSF samples stored for one year was significantly worse than that of serological detection. Not only the fluorescence intensity was significantly reduced, but also the positive rate was significantly reduced. Therefore, if repeated detection is needed, it would be better to subpackage the sample in aliquot before freezing.

\section{Disease characteristics}

Various clinical situations can lower the accuracy of the AQP4-Ab assay (22-24). In addition, some autoimmune 
Table 1 Which patients to test for AQP4-Ab (modified from Whittam et al.) (23)

1. Longitudinally extensive transverse myelitis, especially MRI-revealed increased signal on sagittal T2-weighted MRI extending over 3 or more complete vertebral segments

2. "Idiopathic" acute transverse myelitis lacking other features of MS

3. Severe optic neuritis with poor visual recovery

4. Bilateral simultaneous optic neuritis

5. Longitudinally extensive optic neuritis or involvement of the chiasm; MRI-revealed unilateral or bilateral increased T2 signal or T1 gadolinium enhancement; relatively long lesions extending more than half the distance from orbit to chiasm; and those involving the posterior aspects of the optic nerves or the chiasm

6. Intractable nausea, vomiting or hiccups in the absence of gastro-intestinal pathology

7. Dorsal medullary lesion detected on MRI, especially the area postrema, either small or localized, often bilateral, or contiguous with an upper cervical spinal cord lesion

8. Symptomatic narcolepsy or acute diencephalic clinical syndrome with MRI lesions involving the hypothalamus, thalamus, or periependymal surfaces of the third ventricle

9. Cryptogenic leukoencephalopathy (the leukoencephalopathy cannot be expressed by vascular diseases, infection, metabolic disorder, tumour or any other pathogenesis after system exam)

10. Presumed 'MS' with unexplained severe relapses after starting standard disease-modifying drugs

11. In the above situations, if the initial test was negative, retesting patients at 3-6 months will improve the positive ratio, especially if the initial test was done during clinical remission or treatment with immunosuppression or immediately following plasma exchange

12. MRI-revealed central cord predominance (more than $70 \%$ of the lesion residing within the central grey matter)

13. MRI-revealed longitudinally extensive cord atrophy (sharply demarcated atrophy extending over $>3$ complete, contiguous vertebral segments and caudal to a particular segment of the spinal cord), with or without focal or diffuse T2 signal change involving the atrophic segment on MR imaging

14. MRI-revealed abnormal signals located on periependymal surfaces of the fourth ventricle in the brainstem/cerebellum

15. Optic neuritis with obvious thin RNFL or GCL on OCT

MS, multiple sclerosis; RNFL, retinal nerve fibre layer; GCL, ganglion cell layer; MRI, magnetic resonance imaging; OCT, optical coherence tomography.

diseases such as connective tissue disorders (CTDs) can be combined with NMOSD, and AQP4-Ab can be detected in these entities $(25,26)$. To improve the true positive rate and reduce the rate of false negative and false positive, it is recommended to repeatedly test suspected patients with NMOSD characteristics indicated in Table 1.

(I) As mentioned above, when the sample is collected in the early stage of NMOSD, or after or during the use of high-dose corticosteroids and/or B-cell-depleting agents, the AQP4-Ab may be negative. Some seronegative NMOSD patients initially would gradually become seropositive as the disease progressed $(13,22,27)$. Long et al. (27) retrospectively analyzed 75 seronegative AQP4-Ab patients, 20 of whom were found seropositive when retested the second time.
(II) Samples with lower titres of AQP4-Ab may test negative in a fixed-CBA (17), and samples with highly active AQP4-Ab can destroy AQP4expressing cells (either live-CBA or FACS assay) (17) via the mechanism of antibody dependent cellular cytotoxicity (ADCC), thereby resulting in decreased positive rate.

(III) When the patients with tuberculosis infection, tumors, or natalizumab (NTZ) treatment, the positive AQP4-Ab result should be considered as false positive.

(i) Tuberculosis infection of the CNS could also lead to the production of NMO-IgG/AQP4-Ab. Tuberculosis bacteria and human nerve tissue may have similar AQP4 antigenic epitopes and then induce a cross-reactive response, thereby 
causing false-positive AQP4-Ab (28). The positive rate of NMO-IgG/AQP4-Ab was 76.1$91.3 \%$ in the serum of patients with tuberculous meningitis, regardless of IIF or CBA, and the antibody titre was significantly higher than that of patients with optic neuromyelitis.

(ii) Expression $\mathrm{AQP} 4-\mathrm{Ab}$ might be in tumour patients. Some AQP4-Ab-positive NMOSD cases are associated with tumors, such as those of oesophageal cancer, breast cancer, and renal clear cell carcinoma (29-32). However, there was also the finding of carcinoma in 2 seropositive AQP4-Ab patients who lacked the clinical evidence of an inflammatory CNS demyelinating disorder, suggesting that, in some cases, aquaporin-4-specific IgG may be produced in the course of a tumor immune response (33).

(IV) NTZ can directly interact with the AQP4expressing cells and thereby might cause false-positive AQP4-Ab assay results in some patients (34). In this study, the author showed that NTZ had the ability to strongly bind HEK cells and thus led to a highly positive signal that interfered with the detection of AQP4-Ab in MS patients from a biobank. Anti-AQP4-Ab is mainly IgG1, while NTZ is an IgG4. It was recommended that anti-human IgG1 be used as a secondary antibody to eliminate the NTZ binding problem in these NTZ-treated patients.

(V) AQP4-Ab might be detectable in NMOSD patients concurrent with autoimmune disorders

(i) NMOSD has been shown to be frequently associated with CTDs (e.g., systemic lupus erythematosus or Sjögren syndrome), and AQP4$\mathrm{Ab}$ is usually found in these special populations $(26,35,36)$. CTDs are often associated with wide range, polyclonal B-cell activation, which may result in non-specific reactions. The positive rate of $\mathrm{AQP} 4-\mathrm{Ab}$ in these diseases was almost the same as that in patients with uncomplicated NMOSD, but not in CTDs patients with NMOSD or NMOSD-related neurological symptoms (36). The reason why AQP4-Ab positive NMO/LETM is frequently associated with CTDs is unclear. However, the finding of AQP4-Ab in a subset of patients with CTDs and NMOSD identifies these cases as part of the newly described spectrum of autoimmune
AQP4-channelopathies and suggests that the co-existence of these two disorders in the same patient might reflect a general autoimmune predisposition (26).

(ii) AQP4-Ab might be positive in anti-NMDAR encephalitis (37-39). The diagnosis depends on the clinical manifestation, the treatment response and the prognosis of the diseases. The AQP4$\mathrm{Ab}$ result might be transient $(27,40)$. There are different types of AQP4 antibodies, and only pathogenic antibodies have clinical value in NMOSD. In some cases, the corresponding antibodies might be produced in some AQP4expressing brain areas when exposed to the AQP4 antigen epitope. But it is usually a transient non-pathogenic antibody. With the remission of disease, the immune response will no longer continue, and the antibodies turn negative. The antibody is figuratively compared to a bystander antibody.

(iii) In patients with NMOSD plus myasthenia gravis (MG), the AQP4-Ab results might be positive (41-43). Although most cases had MG prior to NMOSD onset, and most cases had undergone thymectomy, NMOSD can occur first and in patients who have not had their thymus removed. Acetylcholine receptor antibody (AChR-Ab) or AQP4-Ab may be present years before onset of the relevant disease and the titers of AQP4-Ab and AchR-Ab tend to change in opposite directions (42). The incidence of NMOSD plus MG is much higher than that of the general population. There may be a common immunopathological mechanism between them, although this mechanism is not clear at present.

(VI) AQP4 antibody in limited types of NMOSD.

(i) Detection of AQP4-Ab may result in the earlier diagnosis of NMOSD in patients with LETM (44). It is shown that LETM patients with AQP4-Ab positivity have a higher rate of spinal cord expansion and bright spotty lesions $(45,46)$. Furthermore, positive AQP4-Ab predicts a high risk of relapse after the first LETM episode (47) and a higher conversion rate to NMO (48), suggesting a poor prognosis (49).

(ii) Recent researches showed that poorer visual outcomes during acute $\mathrm{ON}$ attacks were found to be more frequent among AQP4-Ab-positive 
patients than among AQP4-Ab-negative patients $(22,27)$. Further research showed that AQP4$\mathrm{Ab}$ was more easily detected in recurrent $\mathrm{ON}$ and bilateral $\mathrm{ON}$ than in unilateral $\mathrm{ON}$ and non-recurrent $\mathrm{ON}$ (11). AQP4-Ab is useful in predicting the severity of the disease course of $\mathrm{ON}$ and the probability of conversion to NMOSD at the first episode of isolated ON (16,50-53).

(iii) Acute brainstem syndrome (ABS) may herald multiple sclerosis (MS) or neuromyelitis optica (NMO), or occur as CIS. AQP4-Ab is an important biomarker for differential diagnosis. Cheng et al. (54) reported that $45.16 \%$ of ABS patients were seropositive for NMO-IgG. In this study, $71.43 \%$ of AQP4-Ab-positive ABS patients converted to $\mathrm{NMO}$, which was much higher than that of the AQP4-Ab-negative ABS patients. AQP4-Ab-positive ABS patients demonstrated a higher IgG index and Kurtzke Expanded Disability Status Scale score (EDSS) than AQP4-Ab-negative ABS patients. Area postrema clinical brainstem symptoms occurred more frequently in AQP4-Ab-positive ABS patients than in AQP4-Ab-negative ABS patients, accompanied by medulla-predominant involvement on MRI.

(VII) For patients with seronegative AQP4-Ab at initial testing but with high clinical suspicion of NMOSD, retesting AQP4-Ab at 3-6 months may yield some additional seropositive cases. The recommended patients for AQP4-testing are shown in Table 1, modified from Whittam et al. (23).

\section{Testing serum and CSF AQP4-Ab together}

It is controversial whether CSF AQP4-Ab needs to be tested at the same time. CSF testing for NMO-IgG/AQP4$\mathrm{Ab}$ is comparatively less sensitive (36,55). If CBA is used for testing, there is little to be gained from routine CSF $\mathrm{AQP} 4-\mathrm{Ab}$ testing in general. The possible matrix effects, such as a higher concentration of ions and a lower amount of total protein compared to serum, would affect antigen/ antibody interactions and even cause false-negative results unless diluted samples are used. However, a recent study by Hao et al. (4) showed that when the AQP4-Ab was detected simultaneously in serum and CSF using CBA, the positive rates were $74.6 \%$ and $44.7 \%$, respectively. The NMO$\mathrm{IgG}$ test results in the same cohort using brain-tissue IIF revealed that the positive rates in serum and CSF were $49.1 \%$ and $17.5 \%$, respectively. Interestingly, there are rare cases in which, AQP4-Ab was positive in CSF but negative in serum. The AQP4-Ab and NMO-IgG of these patients are $6.14 \%$ and $1.75 \%$, respectively (4). The previous study by Klawiter et al. (56) also disclosed that the NMO-IgG/ AQP4-Ab of three patients was detected only in the CSF, but not in the serum. These results indicated that in patients with seronegative NMOSD, testing of CSF is advisable. It would be better to test serum and CSF AQP4-Ab together to improve the positive rate, especially when NMOSD is highly suspected and AQP4-Ab is negative in serum (9).

In our case, even without the result of AQP4-Ab, this patient had met the diagnostic criteria for NMOSD (mentioned above). AQP4-Ab positivity in CSF further supported the NMOSD diagnosis. However, the AQP4$\mathrm{Ab}$ negativity in serum was not consistent with most reports that the AQP4-Ab-positive proportion in serum was higher than that in CSF. Hao et al. $(4,9)$ also showed that there was $6.14 \% \mathrm{AQP} 4-\mathrm{Ab}$ positivity in CSF but negativity in serum. One possible explanation was that RTX affected the expression of AQP4-Ab in serum. Since the molecular weight of rituximab is too large to cross the blood-brain barrier, AQP4-Ab productive B cells would not be removed completely in CSF (57), and there might be a chance at some time point that AQP4-Ab was positive in CSF but negative in serum. The exact mechanism of this phenomenon is not clear and needs further studies. Moreover, the real production location for AQP4-Ab is a pending question to date. In general, the detection of $\mathrm{AQP} 4-\mathrm{Ab}$ in CSF is an important supplement for some seronegative patients, especially for NMOSD or high-risk patients after immunotherapy.

Furthermore, in this case, tuberculosis, tumor and other immune-related diseases that may be positive for AQP4$\mathrm{Ab}$ were excluded. The positive results of $\mathrm{AQP} 4-\mathrm{Ab}$ in the CSF provided a acceptable basis for the diagnosis. In terms of testing methodology, the samples were tested in the laboratory of our hospital within 6 hours after collection, avoiding pre-test status such as long-distance transportation, long-term preservation and repeated freezing and thawing. In addition, three different methods, comprising CBA, ELISA and IIF, were used to detect the samples, making the experimental results more reliable. Besides the positive $\mathrm{AQP} 4-\mathrm{Ab}$ results, the negative $\mathrm{OB}$ results, the clinical characteristics, the MRI features of damage location (more 
than $1 / 2$ length of optic nerve damage, typical brain lesions including corpus callosum injury and paraventricular white matter, among others) were relatively consistent with NMOSD. However, we still cannot completely eliminate diseases such as lymphoma or CTDs, and long-term follow-up, repeated MRI, AQP4-Ab analysis, and tumour marker detection are still very necessary. If possible, the detection of neutrophils, Interleukin-6 (IL-6)/soluble IL-6 receptor (sIL-6R), glial fibrillary acidic protein (GFAP), measles, rubella and varicella zoster virus (MRZ), and so forth, in CSF should be also performed to provide clues for diagnosis, even for a biopsy.

In conclusion, AQP4-Ab is a very important biomarker in the diagnosis of NMOSD. Two methods of detection were recommended to detect $\mathrm{AQP} 4-\mathrm{Ab}$ to increase the positive rate, one of which should be CBA. We constructed an HEK293T cell line transfected with M1-M23-AQP4IgG and identified dominant genetic segments of lentivirus, which was more sensitive than the fixed commercial kit in AQP4-Ab detection. Currently, there are several limitations to making the NMOSD diagnosis directly using an AQP4$\mathrm{Ab}$ result due to the accuracy of different techniques and the use of different sample issues. And it would be better to use both serum and CSF to detect AQP4-Ab. To interpret the results of $\mathrm{AQP} 4-\mathrm{Ab}$ testing, clinicians have to combine all the clinical, imaging and laboratory information.

\section{Acknowledgments}

We acknowledge Miss Minna Zhou and American Journal Expert for their help in language editing.

Funding: This work was supported by grants from the National Natural Science Foundation of China (No. 81273269), the Suzhou Special Project for Clinical Diagnosis and Treatment of Key Diseases (No. LCZX201702), and Key R\&D Plan of Jiangsu Province (Social Development Project) (No. BE2019666).

\section{Footnote}

Provenance and Peer Review: This article was commissioned by the Guest Editors (Hai-Feng Li and Xiangjun Chen) for the series "Laboratory Investigations in Neuroimmunological Diseases and Their Clinical Significance" published in Annals of Translational Medicine. The article has undergone external peer review.

Reporting Checklist: The authors have completed the
Narrative Review reporting checklist. Available at https:// atm.amegroups.com/article/view/10.21037/atm-20-4110/rc

Conflicts of Interest: All authors have completed the ICMJE uniform disclosure form (available at https://atm.amegroups. com/article/view/10.21037/atm-20-4110/coif). The series "Laboratory Investigations in Neuroimmunological Diseases and Their Clinical Significance" was commissioned by the editorial office without any funding or sponsorship. The authors have no other conflicts of interest to declare.

Ethical Statement: The authors are accountable for all aspects of the work in ensuring that questions related to the accuracy or integrity of any part of the work are appropriately investigated and resolved.

Open Access Statement: This is an Open Access article distributed in accordance with the Creative Commons Attribution-NonCommercial-NoDerivs 4.0 International License (CC BY-NC-ND 4.0), which permits the noncommercial replication and distribution of the article with the strict proviso that no changes or edits are made and the original work is properly cited (including links to both the formal publication through the relevant DOI and the license). See: https://creativecommons.org/licenses/by-nc-nd/4.0/.

\section{References}

1. Wingerchuk DM, Banwell B, Bennett JL, et al. International consensus diagnostic criteria for neuromyelitis optica spectrum disorders. Neurology 2015;85:177-89.

2. Damato V, Evoli A, Iorio R. Efficacy and Safety of Rituximab Therapy in Neuromyelitis Optica Spectrum Disorders: A Systematic Review and Meta-analysis. JAMA Neurol 2016;73:1342-8.

3. Lennon VA, Wingerchuk DM, Kryzer TJ, et al. A serum autoantibody marker of neuromyelitis optica: distinction from multiple sclerosis. Lancet 2004;364:2106-12.

4. Hao HJ, Liu R, Liu LL, et al. Analysis the patients with AQP-4 Antibody/ NMO-IgG positive NMOSD. Chinese Journal of Clinicians (electronic version) 2015;9:3844-8.

5. Bradl M, Misu T, Takahashi T, et al. Neuromyelitis optica: pathogenicity of patient immunoglobulin in vivo. Ann Neurol 2009;66:630-43.

6. Höftberger R, Sabater L, Marignier R, et al. An optimized immunohistochemistry technique improves NMO-IgG detection: study comparison with cell-based assays. PLoS 
One 2013;8:e79083.

7. Ruiz-Gaviria R, Baracaldo I, Castañeda C, et al. Specificity and sensitivity of aquaporin 4 antibody detection tests in patients with neuromyelitis optica: A meta-analysis. Mult Scler Relat Disord 2015;4:345-9.

8. Siritho S, Apiwattanakul M, Nakashima I, et al. Features of anti-aquaporin 4 antibody-seronegative Thai patients with neuromyelitis optica spectrum disorders: a comparison with seropositive cases. J Neurol Sci 2014;341:17-21.

9. Waters PJ, McKeon A, Leite MI, et al. Serologic diagnosis of NMO: a multicenter comparison of aquaporin-4-IgG assays. Neurology 2012;78:665-71; discussion 669.

10. Kim W, Lee JE, Li XF, et al. Quantitative measurement of anti-aquaporin-4 antibodies by enzyme-linked immunosorbent assay using purified recombinant human aquaporin-4. Mult Scler 2012;18:578-86.

11. Jiao Y, Fryer JP, Lennon VA, et al. Updated estimate of AQP4-IgG serostatus and disability outcome in neuromyelitis optica. Neurology 2013;81:1197-204.

12. Majed M, Fryer JP, McKeon A, et al. Clinical utility of testing AQP4-IgG in CSF: Guidance for physicians. Neurol Neuroimmunol Neuroinflamm 2016;3:e231.

13. Marignier R, Bernard-Valnet R, Giraudon P, et al. Aquaporin-4 antibody-negative neuromyelitis optica: distinct assay sensitivity-dependent entity. Neurology 2013;80:2194-200.

14. Fujihara K, Sato DK. AQP4 antibody serostatus: Is its luster being lost in the management and pathogenesis of NMO? Neurology 2013;81:1186-8.

15. Waters P, Reindl M, Saiz A, et al. Multicentre comparison of a diagnostic assay: aquaporin-4 antibodies in neuromyelitis optica. J Neurol Neurosurg Psychiatry 2016;87:1005-15.

16. Waters PJ, Pittock SJ, Bennett JL, et al. Evaluation of aquaporin-4 antibody assays. Clin Exp Neuroimmunol 2014;5:290-303.

17. Yang J, Kim SM, Kim YJ, et al. Accuracy of the Fluorescence-Activated Cell Sorting Assay for the Aquaporin-4 Antibody (AQP4-Ab): Comparison with the Commercial AQP4-Ab Assay Kit. PLoS One 2016;11:e0162900.

18. Potokar M, Stenovec M, Jorgačevski J, et al. Regulation of AQP4 surface expression via vesicle mobility in astrocytes. Glia 2013;61:917-28.

19. Mader S, Lutterotti A, Di Pauli F, et al. Patterns of antibody binding to aquaporin-4 isoforms in neuromyelitis optica. PLoS One 2010;5:e10455.

20. Jarius S, Wildemann B. Effect of storage conditions and freeze/thaw cycles on aquaporin-4 antibody (NMO-IgG) serum levels. Clin Chem Lab Med 2011;49:2121-2.

21. Yang XG, Yao HY, Fan RX, et al. Effect of storage condition and freeze/thaw cycles on aquaporin-4 antibody in serum and cerebral spinal fluid. The Journal of Practical Medicine 2016;32:1515-7.

22. Waschbisch A, Atiya M, Schaub C, et al. Aquaporin-4 antibody negative recurrent isolated optic neuritis: clinical evidence for disease heterogeneity. J Neurol Sci 2013;331:72-5.

23. Whittam D, Wilson M, Hamid S, et al. What's new in neuromyelitis optica? A short review for the clinical neurologist. J Neurol 2017;264:2330-44.

24. Kim SM, Kim SJ, Lee HJ, et al. Differential diagnosis of neuromyelitis optica spectrum disorders. Ther Adv Neurol Disord 2017;10:265-89.

25. Shahmohammadi S, Doosti R, Shahmohammadi A, et al. Autoimmune diseases associated with Neuromyelitis Optica Spectrum Disorders: A literature review. Mult Scler Relat Disord 2019;27:350-63.

26. Jarius S, Jacobi C, de Seze J, et al. Frequency and syndrome specificity of antibodies to aquaporin-4 in neurological patients with rheumatic disorders. Mult Scler 2011;17:1067-73.

27. Long Y, Liang J, Zhong R, et al. Aquaporin-4 antibody in neuromyelitis optica: re-testing study in a large population from China. Int J Neurosci 2017;127:790-9.

28. Sun QS, Liu JX, Feng YQ, et al. The detection and specific study of NMO-IgG/anti-AQP4 antibody. Chinese Journal of Clinicians (electronic version) 2010;4:2147-52.

29. Kon T, Ueno T, Suzuki C, et al. Aquaporin-4 antibody positive neuromyelitis optica spectrum disorder associated with esophageal cancer. J Neuroimmunol 2017;309:38-40.

30. Liao W, Li C, Tang Y, et al. Aquaporin-4 antibody positive short transverse myelitis associated with breast cancer. Mult Scler Relat Disord 2019;30:119-22.

31. Jin H, Hao H, Nan D, et al. Neuromyelitis optica spectrum disorder coincident with renal clear cell carcinoma: A case report. Medicine (Baltimore) 2019;98:e14229.

32. Berger B, Hottenrott T, Rauer S, et al. Screening for onconeural antibodies in neuromyelitis optica spectrum disorders. BMC Neurol 2017;17:5.

33. Pittock SJ, Lennon VA. Aquaporin-4 autoantibodies in a paraneoplastic context. Arch Neurol 2008;65:629-32.

34. Cohen M, De Sèze J, Marignier R, et al. False positivity of anti aquaporin-4 antibodies in natalizumab-treated patients. Mult Scler 2016;22:1231-4.

35. Jayarangaiah A, Sehgal R, Epperla N. Sjögren's syndrome 
and neuromyelitis optica spectrum disorders (NMOSD)-a case report and review of literature. BMC Neurol 2014;14:200.

36. Jarius S, Wildemann B. Aquaporin-4 antibodies (NMO-IgG) as a serological marker of neuromyelitis optica: a critical review of the literature. Brain Pathol 2013;23:661-83.

37. Ran Y, Wang L, Zhang F, et al. Anti-NMDAR encephalitis followed by seropositive neuromyelitis optica spectrum disorder: A case report and literature review. Clin Neurol Neurosurg 2017;155:75-82.

38. Qin K, Wu W, Huang Y, et al. Anti-N-methyl-Daspartate receptor(NMDAR) antibody encephalitis presents in atypical types and coexists with neuromyelitis optica spectrum disorder or neurosyphilis. BMC Neurol 2017;17:1.

39. Luo JJ, Lv H, Sun W, et al. Anti-N-methyl-d-aspartate receptor encephalitis in a patient with neuromyelitis optica spectrum disorders. Mult Scler Relat Disord 2016;8:74-7.

40. Kitley J, Woodhall M, Leite MI, et al. Aquaporin-4 antibody isoform binding specificities do not explain clinical variations in NMO. Neurol Neuroimmunol Neuroinflamm 2015;2:e121.

41. Wu D, Cui Y, Hou, et al. Characteristic and pathogenesis of two patients with myasthenia gravis associated with neuromyelitis optica. Chin J Neuromed 2017;16:82-5.

42. Leite MI, Coutinho E, Lana-Peixoto M, et al. Myasthenia gravis and neuromyelitis optica spectrum disorder: a multicenter study of 16 patients. Neurology 2012;78:1601-7.

43. Jarius S, Paul F, Franciotta D, et al. Neuromyelitis optica spectrum disorders in patients with myasthenia gravis: ten new aquaporin-4 antibody positive cases and a review of the literature. Mult Scler 2012;18:1135-43.

44. Carnero Contentti E, Hryb JP, Morales S, et al.

Longitudinally extensive transverse myelitis immunemediated in aquaporin-4 antibody negative patients: Disease heterogeneity. J Neurol Sci 2017;373:134-7.

45. Chee CG, Park KS, Lee JW, et al. MRI Features of Aquaporin-4 Antibody-Positive Longitudinally Extensive Transverse Myelitis: Insights into the Diagnosis of Neuromyelitis Optica Spectrum Disorders. AJNR Am J Neuroradiol 2018;39:782-7.

46. Murchison A, Kitley J, Leite MI, et al. Predictive value of MRI parameters in severity and recovery of first-episode myelitis in aquaporin-4 antibody disease. J Neurol Sci 2015;355:49-53.

47. Weinshenker BG, Wingerchuk DM, Vukusic S, et al. Neuromyelitis optica IgG predicts relapse after longitudinally extensive transverse myelitis. Ann Neurol 2006;59:566-9.

48. Wang J, Li X, Zhang DQ, et al. Quantitative analysis of aquaporin-4 antibody in longitudinally extensive transverse myelitis. J Neuroimmunol 2015;278:26-9.

49. Jiao Y, Fryer JP, Lennon VA, et al. Aquaporin 4 IgG serostatus and outcome in recurrent longitudinally extensive transverse myelitis. JAMA Neurol 2014;71:48-54.

50. Lai C, Tian G, Takahashi T, et al. Neuromyelitis optica antibodies in patients with severe optic neuritis in China. J Neuroophthalmol 2011;31:16-9.

51. Kezuka T. Optic neuritis--immunological approach to elucidate pathogenesis and develop innovative therapy. Nippon Ganka Gakkai Zasshi 2013;117:270-91; discussion 292.

52. Wingerchuk DM, Lennon VA, Lucchinetti CF, et al. The spectrum of neuromyelitis optica. Lancet Neurol 2007;6:805-15.

53. Li H, Wang Y, Xu Q, et al. Features of anti-aquaporin 4 antibody-seropositive Chinese patients with neuromyelitis optica spectrum optic neuritis. J Neurol 2015;262:2293-304.

54. Cheng C, Jiang Y, Lu X, et al. The role of anti-aquaporin 4 antibody in the conversion of acute brainstem syndrome to neuromyelitis optica. BMC Neurol 2016;16:203.

55. Takahashi T, Fujihara K, Nakashima I, et al. Antiaquaporin-4 antibody is involved in the pathogenesis of NMO: a study on antibody titre. Brain 2007;130:1235-43

56. Klawiter EC, Alvarez E 3rd, Xu J, et al. NMO-IgG detected in CSF in seronegative neuromyelitis optica. Neurology 2009;72:1101-3.

57. Ferreri AJ, Cwynarski K, Pulczynski E, et al. Chemoimmunotherapy with methotrexate, cytarabine, thiotepa, and rituximab (MATRix regimen) in patients with primary CNS lymphoma: results of the first randomisation of the International Extranodal Lymphoma Study Group-32 (IELSG32) phase 2 trial. Lancet Haematol 2016;3:e217-27.

Cite this article as: Xue Q, Cao S, Rui Q. Reflections from a NMOSD case with serum AQP4-Ab negativity but CSF positivity: narrative review of how to interpret AQP4-Ab test results. Ann Transl Med 2023;11(7):286. doi: 10.21037/atm-204110 


\section{Appendix 1 In-house CBA}

Our laboratory constructed an HEK293T cell line transfected with lentivirus containing M1 and M23-AQP4 sequence. M1-M23-AQP4-IgG identified dominant genetic segments of lentivirus pLV-CMV-AQP4-M1-M23EGFP-Puro. HEK293 cells transfected with the negative control lentivirus pLV-CMV-NC-EGFP-Puro were used as a control. The lentivirus transfection rate of HEK293 cells was detected and identified by flow cytometry. Cells with a transfection rate of more than $95 \%$ were collected, and cell smear slides were made, dried, and frozen at $-80^{\circ} \mathrm{C}$. Before detection, the slides were removed and fixed. To determine the appropriate dilution, AQP4-Ab positive serum and Cy3-labelled donkey anti-human IgG (Jackson immune, USA) were diluted at different concentrations and then immunofluorescence staining was used. Finally, we established a monoclonal HEK293 cell line that stably expressed M1-M23-AQP4 and made long-term, preservable smeared cell slides using the Jilt piece technique. Using this kind of frozen slide, the test results were stable even after being frozen for 16 weeks (data not shown), which suggested that this technique with high stability might satisfy the clinical requirement at any time. To verify the reliability of the system, from April 2015 to June 2016, our laboratory tested 110 samples from different patients, and the results are shown in Table S1. In this study, only 1 out of 24 patients with LETM was positive for serum AQP4$\mathrm{Ab}$ (titre 1:8); 3 of 18 patients with optic neuritis were seropositive, with titres ranging from 1:4 to 1:8. All 22 patients with other neuroimmune diseases and 16 healthy controls were tested negative. The positive rate of CBA was $73.33 \%(22 / 30)$, which was higher than that of ELISA $63.33 \%(19 / 30)$ (Table S1). This result is consistent with previous reports regarding M23-based CBA $(4,10)$. In this group of NMOSD patients, the samples were also sent to Euroimmun, and they used IIF to test the expression of NMO-IgG in the samples. The NMO-IgG positive rate was $60 \%(18 / 30)$, which seemed to be lower than that of AQP4-Ab with CBA of $73.33 \%$ (22/30).

Table S1 The essential information of 110 enrolled patients and the results of AQP4-Ab detection.

\begin{tabular}{|c|c|c|c|c|c|}
\hline & $\mathrm{HC}, \mathrm{n}=16$ & NMOSD, $n=30$ & LETM, $\mathrm{n}=24$ & ON, $n=18$ & ONID, $n=22$ \\
\hline Gender (Female/male) & $8 / 8$ & $24 / 6$ & $12 / 12$ & $10 / 8$ & $12 / 10$ \\
\hline $\begin{array}{l}\text { ELISA-AQP4-Ab (U/L)/ } \\
\text { Positive AQP4-Ab (\%) }\end{array}$ & $0.68 \pm 0.12 / 0$ & $25.89 \pm 4.60 / 33.33(19 / 30)$ & $1.75 \pm 0.15 / 4.17(1 / 24)$ & $1.26 \pm 0.16 / 16.67(3 / 18)$ & $1.47 \pm 0.18 / 0$ \\
\hline $\begin{array}{l}\text { CBA-AQP4-Ab/Positive } \\
\text { AQP4-Ab (\%) }\end{array}$ & - & $1: 32-1: 1024 / 73.33(22 / 30)$ & $1: 2-1: 8$ & $1: 4-1: 8$ & $0-1: 2$ \\
\hline
\end{tabular}

NMOSD, neuromyelitis optic spectrum disease; LETM, Iongitudinally extensive transverse myelitis; ON, optic neuritis; ONID, other neural system autoimmune diseases; $\mathrm{HC}$, healthy control. 